\title{
ON
}

\section{THE USE OF ATROPINE IN CHOLERA.}

\author{
BY \\ T. LAUDER BRUNTON, M.D., \\ F.R.C.P., F.R.S., LL.D. HON. ABER. \\ Received April 26th-Read June 13th, 1893.
}

I HAVE to ask the indulgence of the Society for bringing before it a paper founded upon only one case. My excuse for doing so is that the case is one for which I have been obliged to wait nearly twenty years, and the probability of a return of cholera during the present year is so great that it is well to discuss all means of treatment which are likely to be useful in the disease before it actually begins to spread again, either in this country or on the Continent.

In 1873, in a paper read before the British Association at Bradford, I pointed out the great likeness which exists between the symptoms of cholera and those produced by poisoning with muscarine; and I then suggested that as atropine completely antagonised the action of muscarine, and prevented death from what would otherwise have been a fatal dose, it might possibly be useful in the treatment of cholera. At that time the pathology of infective diseases was not understood as it is now. One could not point to any organism as the cause of cholera, nor did our knowledge allow of any distinction being drawn between pathogenic microbes and the poisons which they produce. I did not, therefore, attempt to discuss more closely the nature of 
the poison. While the infective nature of cholera and its transmission from place to place seemed to point to a microbe as its cuuse, yet Lewis and Cunningham, ${ }^{1}$ in a paper published in the year subsequent to mine, announced that they had failed to find any microbe in the blood or tissues, but had succeeded in producing symptoms of gastro-enteric irritation by the injection of boiled cholera dejecta into the blood of animals. These observations of Lewis and Cunningham appeared to show that the cholera dejecta contained the poisonous substance quite apart from any living organism which might be present in them. Since that time the researches of Koch have shown that the so-called comma bacillus is the organism most frequently found in cases of cholera, but according to Cunningham ${ }^{2}$ other varieties of bacillus may produce symptoms indistinguishable from those of cases where the true comma bacillus is found. We may, therefore, regard it as certain that a micro-organism is the cause of the disease, although it is quite possible that this may not always be of the samecharacter; and it is unnecessary to discuss this subject further at present, inasmuch as the subject we have to deal with to-night is not so much the effect of drugs upon the organism which gives rise to the disease as the treatment of the symptoms which the patient presents.

It is now, I think, generally agreed that these symptoms are not caused by bacilli themselves which remain in the intestine, and do not pass into the tissues, but are rather due to some poison which these bacilli produce, and which, after absorption into the circulation, acts upon various parts of the organism. When we come to inquire into the nature of this poison we find that opinions are divided about it as well as about the bacillus which generates it. According to Hueppe it is a substance resembling peptones in its physical and chemical reactions, but differing from them in this respect, that its activity

1 "Nature of Agent or Agents producing Cholera," Calcutta, 1874.

2 D. D. Cunningham, vide "The Bacteriology of Cholera," by A. Crombie, M.D., ' Practitioner,' vol. xlvii, p. 330. 
is destroyed by boiling. It is evident from this statement that the substance regarded by Hueppe as the poison is not the same as that with which Lewis and Cunningham were dealing in their experiments with fresh cholera dejecta. I am therefore at the present moment, notwithstanding all the work which has been done upon cholera, unable to speak much more definitely about the poison than I could twenty years ago, and I may therefore fairly quote from my paper read at the British Association at Bradford, and say, "The cause of cholera is now generally admitted to be a poison of some sort, which can be conveyed about from place to place and transmitted from one person to another, through the medium of the evacuations, which either get into water and are drunk, or become dry and are taken into the mouth and nostrils in the shape of dust. Some even yet are inclined to hold that cholera results rather from peculiar atmospheric and other conditions than from the presence of a specific poison; but the fact that the disease may be conveyed from one infected locality to numerous others by a single individual, breaking out where he has stopped, and passing over those places which he has only travelled through, although those may present apparently identical conditions of air, sea, and water, shows conclusively that an outbreak of the pestilence cannot be due to these latter circumstances alone. Nor will the mere presence of the poison always produce cholera, for those who are exposed to contagion do not all become affected, and even those who have swallowed cholera stools, in which the poison is supposed to be present in its most concentrated form, have sometimes escaped with impunity. It would appear that two conditions are required, viz. the presence of the poison and the existence of a proper soil for its development. In other words, it would seem that the poison does not produce its usual effects even when it has entered the system, unless the blood and tissues are in such a state that it may act upon them. The nature of this state we cannot exactly define, but its 
presence seems to be due in great measure to those conditions of atmosphere and soil which some assert to be the immediate cause of the disease, but which in reality only predispose to it.

"Without entering into this question at any greater length, I shall assume that cholera is caused by a specific poison acting upon an organism which has become in some way or other susceptible to its influence. The effects of the poison upon the body may be summed up in few words. It produces irritability of the digestive canal, immoderate secretion from the intestines, and lessened circulation both through the lungs and the body. Bearing in mind these actions, it is perfectly easy for anyone to deduce from them all the symptoms which are observed in the state of cholera collapse. From the irritability of the stomach and intestines there is constant vomiting and purging. The secretion from them is so profuse that the whole intestinal canal is speedily washed clean out; the stools are no longer feculent nor even tinged with bile, but consist of the secretion alone, pure and unmixed, and resembling rice water in appearance. The blood is thus drained of its fluid parts, and the consequence of this is intense thirst, which adds greatly to the sufferings of the patient. The blood itself, instead of coursing rapidly through the vessels as it does in health, stagnates in the great veins of the thorax and abdomen; the left side of the heart, instead of receiving from the lungs a full supply of well-aërated blood which it would propel through every part of the body, receives only a scanty driblet which leaves it almost collapsed; the arteries which proceed to the body are so empty that when they are cut across hardly a drop of blood flows from them; and even when a tube is passed through the carotid artery and aorta right up to the sigmoid valves of the heart, as was done by Dieffenbach, no blood can be drawn from it. The warm blood from the interior of the body, which usually circulates in the vessels near the surface, imparting to it the plumpness, warmth, and rosy 
hue of health, stagnates in the abdominal veins, and leaves the skin shrunken, pale, and cold; while that in the interior of the body, being no longer cooled by circulation near the surface, becomes hotter and hotter, till the internal temperature of the unfortunate patient is higher than it usually is in high fever, though his skin and breath are cold as ice. The blood which fills the small cutaneous veins, being no longer driven forward by fresh supplies from the arteries, becomes completely deoxidised and black, imparting to the surface a livid hue. So dark does the blood become that it assumes the colour of bilberry juice, and the colouring matter leaves the corpuscles and tinges the serum. It still retains its power to take up oxygen and give off carbonic acid, but notwithstanding this it passes so slowly through the pulmonary vessels that only about one third of the usual quantity of carbonic acid is given off from the lungs, and little oxygen being taken in, there is a distressing feeling of want of breath. The voice at the same time is hoarse, low, and weak, but this seems to be simply a consequence of the general exhaustion of the patient.

"Such are the symptoms of cholera, all rising from disturbance of the circulation and excessive intestinal secretion. The remedy we seek must, therefore, be one which has the power of removing these conditions. It may be thought that the only way to do this is to eliminate from the body the poison which is producing these results, and that so long as it is still circulating in the blood any remedy which is simply intended to counteract its effects will be administered in vain. But the researches of Fraser and others on antagonism have shown us that the elimination of a poison is not required in order to prevent its injurious or fatal action, for the administration of an antidote will deprive it of its hurtful power, and as it is with other poisons, so may it be with cholera."

If we compare these symptoms with those of poisoning by muscarine, as described by Schmiedeberg, we find a remarkable resemblance between them. They are profuse secre- 
tion of tears and saliva, very violent retching and vomiting, and diarrhœa with much griping. There is contraction of the pupil, very frequent and laboured breathing, and in some animals a rapid, and in others a slow pulse, muscular weakness, cessation of the respiration, and death. On making some experiments as to the cause of dyspnœa, I came to the conclusion that the vessels of the lung were contracted by the drug, so that the blood could not readily pass from the right to the left side of the heart. After the administration of muscarine I saw the right side of the heart become distended, the lungs become pale, and the left ventricle become contracted. The administration of a small dose of atropine caused a complete change in these appearances. The right ventricle emptied itself, the lungs became rosy, and the left side of the heart again became filled. The effects led me to think that atropine might be a useful remedy in cholera, but I had no opportunity of testing this myself until last August, when two cases arrived in London from Hamburg and were brought to St. Bartholomew's Hospital. One of these was a man, Gustave Rosenbaum, æt. 35, who left Hamburg on August 27th, apparently quite well, but was very sick and suffered from diarrhœa during a rough passage. He landed at Harwich on the morning of August 29th, and came on to London the same morning. He seemed fairly well during theday. During the evening he was taken ill, had diarrhœa, with pains across the back and cramps down the legs, chiefly in the right. $\mathrm{He}$ had diarrhœa all night. The motions were loose but coloured. On the morning of the 30th he vomited, had no diarrhœa after 11 a.m., but suffered much from pain across the back, and was brought to St. Bartholomew's late in the afternoon. Having been summoned to an urgent case of pneumonia at some distance off in the country, I did not see the patient, but I heard he was to come in, and I discussed the treatment with the house physician. When admitted he had a sallow, grey look, his eyes were sunken, pupils equal, not contracted. His pulse was scarcely perceptible at the wrist, 148 , very 
feeble; the respiration was sighing, 56 ; the temperature $95 \cdot 8^{\circ}$. The first sound was distinct at the apex, the second sound was not heard, and both sounds were very feeble at the base. The arms were cold, and the fingers were cold and dusky. He was ordered Liquor Hydrarg. Perchlor. half a drachm, spt. of chloroform 5 minims, and water up to an ounce every four hours, to act as an intestinal disinfectant. At ten o'clock he was very restless, complained of great pain in his back and the calves of his legs, and was ordered the mixture every two hours. At 1.20 a.m. on the 31st he was still very restless ; his pulse was perceptible at the wrist, 160 per minute; the respirations were 40. The bowels were open twice, once into the bedpan and once into the bed. The motions were hardly coloured at all. He passed a small quantity of urine, which contained a small cloud of albumen, and was acid and high-coloured. At 2.15 the bowels were open, then again at 2.45, and again at 3 . At 3.30 he asked for the bedpan, and then suddenly collapsed and died. Although the motions up to the last hardly presented the typical ricewater appearance, the comma bacillus was found in large numbers by Mr. Cautley in the intestine and Dr. Klein confirmed the observation.

On post-mortem examination the mucous membrane of the intestines was rather pale, with a few reddish patches on the lower part of the small intestine. The blood in the veins of the neck and of the heart was thicker and blacker than usual. The spleen was normal, and there was only one mesenteric gland at all enlarged. The other organs were merely normal.

At the same time as this patient his daughter, Casperina R-, æt. 3, was brought into the hospital.

She was taken ill upon the 24th of August, with spots on her face and diarrhœa. From this she appeared to recover, but remained somewhat languid. On the 27th she left Hamburg. She was sick on board ship, but had little diarrhœa, and on August 29th, when she came to town, was fairly well. On the morning of the 30th she 
was taken ill with frequent diarrhœa and violent vomiting. The motions were slightly coloured, and there was no pain. On admission to St. Bartholomew's on the evening of the 30th she was dusky, with pale lips and sunken eyes; the pupils were equal and of moderate size, the conjunctivæ injected. The legs and arms were cold, clammy, and dusky. The tongue was moist. The pulse was 143, feeble. The respiration was 39 , sighing, the temperature $97^{\circ}$. The impulse of the heart was feeble, the first sound absent at the apex and the second at the base.

The child was ordered milk, soda water and brandy, Liq. Hydrarg. Perchlor. mxv, Spt. Chloroform. mij, and water up to half an ounce every two hours. At eleven o'clock the child was not so well as on admission. The bowels had been open three times, and the motions were of a pale brown colour, considerable in quantity, and contained no solid matter. At 1.30 the extremities were cold, lips very pale, pulse 152 , easily counted; resp. 24, long-drawn and sighing; no more diarrhœa. On August 31 st I saw the child at 9 a.m. ; the pulse was better, hands warmer, no diarrhœa. At 1 p.m. the pulse was feebler, the eyes were sunken and glassy, no urine had been passed. The lips were not so blue; the respiration was 20 , not so sighing; pulse 124. At 5.30 the child was in the same condition, but the hands were colder; the pulse was 140. One two-hundredth of a grain of atropine was then given subcutaneously. In ten minutes the child appeared flushed, the pulse went up to 160, the respiration to 24, and was not so sighing. For a short time after the injection the child slept quietly. The bowels were not open, and no more urine was passed. There was some sickness, and in consequence of this the Liq. Hydrarg. Perchlor. was stopped, and Bism. Subnit. was given instead. Just before midnight there was more vomiting and sighing respiration, and a quarter of a grain of calomel was ordered every two hours. At 3.45 the respirations had sunk to 12 , and they were long-drawn and sighing. The pulse was 140 and the surface very cold. One four- 
hundredth of a grain of atropine was then injected subcutaneously. In a quarter of an hour the hands became rather warmer, the pulse remained at 140 , but the respiations rose to 18 per minute. Small enemeta of water, half an ounce, were also given. During September 1st the condition varied a good deal, but the respiration did not again sink so low as before, although towards evening it fell to 15 . There was considerable sickness, but only one motion in the early morning. On the 2nd the child was in the same condition, passed a greenishcoloured motion with some solid matter in the early morning. From this time the child steadily improved until she left the hospital on September 8th, completely recovered.

In this case the injection of atropine acted as a powerful stimulant when the respiration appeared about to fail, and increased the rate of breathing and the warmth of the surface. It did not appear to have any influence upon the retching, vomiting, or diarrhœa. This is precisely what one would expect from the experiments which Dr. Pye-Smith and I conducted a number of years ago, and which we reported to the British Association in 1874, 1875, and 1876. In the first series of these experiments Dr. West was associated with us in the committee of the Association. The whole of these papers were reprinted, and the pathology and treatment of cholera in general were discussed by Dr. Pye-Smith and myself in the 'Practitioner' for November, 1884, and in subsequent numbers.

In summing up our conclusions regarding treatment, we observed that two desiderata in the treatment of cholera were (1) an antiseptic to destroy the cholera bacillus and prevent it from forming poisons in the intestine, and (2) a remedy which would antagonise the action of the poison after its absorption from the intestine.

In the treatment of the case which I have described, the perchloride of mercury and calomel were used for the purpose of disinfecting the intestine, the atropine was given for the purpose of antagonising the action of the 
cholera poison upon the circulation and respiration. I have already mentioned that we do not yet know the exact nature of the cholera poison; and it seems not at all unlikely that there are more than one, because, as I have already pointed out, Hueppe and Cunningham appear to have had different poisons, both of which they had obtained from cholera dejecta or cultivations from them, and $M$. Villiers obtained from the intestinal contents of cholera patients an alkaloid which had a marked action upon the heart, sometimes slowing it greatly, and at other times greatly increasing its rapidity. Claude Bernard has stated that in some cholera patients slowing of the heart was observable for some days before the attack, and this rather points to the presence in the circulation of a poison having an action like muscarine. It is a well-known fact that some mushrooms are intensely poisonous, while others are not, but occasionally some species become poisonous when grown under certain conditions. It seems not impossible, therefore, that the cholera bacilli, even if we suppose them to be originally of the same nature, may not only undergo variations in their appearance, but may form poisons according to the conditions which they meet in the intestine. Amongst these it is by no means improbable that muscarine itself may actually be formed, inasmuch as Brieger has shown it to be not an uncommon product of albuminous decomposition during putrefaction; and, if so, atropine is likely to be useful in certain cases. But atropine cannot be looked upon at all as a universal remedy for cholera. It is not likely to affect the profuse intestinal discharge, and will probably, therefore, do but little good in cases where this is very profuse. But in such cases as those two which I have described, where the intestinal discharge was scanty and the vascular symptoms were pronounced, atropine may be useful, and undoubtedly in the case of the little girl, Casperina Rosenbaum, a marked improvement appeared to follow immediately upon its injection, although a relapse occurred subsequently. Before concluding I may mention some interesting obser- 
vations of Alt, who has found that not only is the venom of snakes excreted from the stomach when injected subcutaneously, but that toxalbumins of cholera are excreted in a similar manner, and that washing out the stomach may also be useful as an adjunct to other treatment. In a paper that I wrote in the 'British Medical Journal' I pointed out the probable utility of washing the stomach out in case of a snake-bite, but Alt's observations were only published a month or two ago, or I should have tried the effect of washing out the stomach in the case of Casperina Rosenbaum, either with water, dilute saline solution, or with a weak solution of permanganate of potash.

The general treatment of cholera is, however, too wide a question to go into here, and I must be content with simply pointing out the possible use of atropine as a cardiac and respiratory stimulant in some cases of the disease.

In conclusion, I desire to acknowledge the careful attention of Dr. Batten, house physician, and to thank him for his laborious observation of the cases.

St. Bartholomew's Hospital (August 30th, 1892).Casperina R-, æt. 3.

History.-Child taken ill six days ago, had spots on her face, had diarrhœa, after medicine got better. Left Hamburg on Saturday, August 27th, three days ago. Somewhat languid after the diarrhœa. One hundred and eleven on board ship. No diarrhøa to speak of. Fairly well on August 29th, when she came to town. Yesterday evening child slept fairly well. This morning child first taken ill. Had frequent diarrhœa, motions slightly coloured. Child vomited after everything given except Mellin's food ; no pain.

Family history.-Mother well. Father choleraic symptoms. Only child. Past illness.-Always healthy.

Present condition.-Dusky, eyes sunken, lips pale, tongue moist. Pulse 143, feeble, easily countable at the wrist. Respiration sighing, 39, long-drawn inspirations 
at times. Temp. 97. Eyes very much sunken, pupils equal, moderate size, conjunctiva injected. Arms : hands cold, clammy, dusky colour. Chest : breath-sounds natural. Heart : impulse feeble, absence of first sound at apex and second sound at base. Legs cold, clammy, blue. Urine. Abdomen resonant, no tenderness; liver not enlarged.

11.10. - Child not so well as on admission. Bowels opened three times since admission; motions pale brown colour, no solid matter, a good deal in quantity. No vomiting. Ordered milk, soda water and brandy, and Liq. Hydrarg. Perchlor., mxv ; Spt. Chloroform., mij ; Aq. ad $\frac{1}{2}$ oz., 2 dis hor.

1.30.-Extremities cold, pulse easily countable at wrist, 152. Resp. 24, sighing, long-drawn; no more diarrhœa ; lips very pale.

August 31st, 9 a.m.-Pulse better, hands warmer, no diarrhœa.

1 p.m.-Pulse not so good as the morning. Eyes sunken. Child taking not so well. No urine passed. Lips not blue. Eyes glassy. Resp. 20, not so sighing. Pulse 124.

5.30.-Child remains in same condition. Hands colder. About 1.30 passed some urine; no motion. Pulse 140. Child will not take readily; injection of $\frac{1}{200} \mathrm{gr}$. of atropine subcutaneously.

5.40.-Child appears flushed. Pulse 160. Resp. 24, not so sighing.

7.30.-The last dose of Hydrarg. Perchlor. was given at 4.30. It was not repeated at 6.30 on account of the sickness. Orảered Bismuth. Subnit., gr. iss ; Sod. Bicarb., gr. iiss ; Spt. Chlorof., miiss ; Aq. Arni, zij p. r. n. for the sickness. Child vomited about 1 oz. Pulse 132. Resp. 20. Not so flushed. Child apparently slept quietly for a short time after the injection. Hands fairly warm. Bowels not opened. No more urine passed. 10.15.-Child vomited again. Pulse 140, resp. 24.

September 1st, 12.15.-Child vomited again at 11.45. 
Pulse 120, respiration sighing. Ordered calomelanos gr. $\frac{1}{4}$ secundis horis.

1.20.- Not vomited again, hands rather colder. Pulse not so good, 120.

3.45.-Child vomited again at 3.15 ; about 5 oz. of milk given. Resp. 12, long-drawn; pulse 140. Injection of $\frac{1}{400} \mathrm{gr}$. of atropine.

4.- -Hands rather warmer. Pulse 140, resp. 18. Enemata of $\frac{1}{2}$ oz. of water.

September 1st, 9.50.-During the past six hours the child has taken only $6 \mathrm{dr}$. of milk and soda water; the enema given at four o'clock was retained as well as the one given

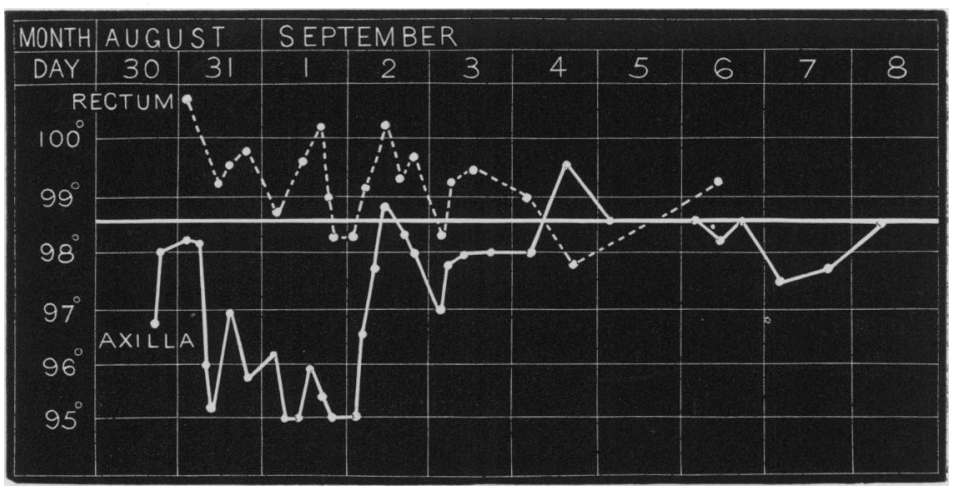

an hour later. The one given at 6.15 was returned with loose light-coloured motion. The child seemed brighter this morning. Pulse not so good, 120, fairly regular; hands cold, feet warm. Respiration more sighing for last hour, 20. No vomiting since 3.15.

12.45.-Vomited twice since 9.50. Child seems brighter. Pulse 140.

5 p.m.-Pulse much better, 108, fair volume ; resp. 20. No vomiting since 12.30 . Taken $5 \mathrm{oz}$. of milk and soda water, $1 \frac{1}{2} \mathrm{dr}$. of brandy, two enemata of $\frac{1}{2} \mathrm{oz}$. each. Passed over $1 \mathrm{oz}$. of urine at 10.50 ; urine acid, very faint trace of albumen.

9.30.-Pulse not quite so good, 120. Child vomited vol. LXXVI. 
once this evening and retched for some time afterwards. The child slept afterwards. Taken Oss. in last twelve hours. Child rather more restless. Had arrowroot water this evening, vomited this evening after it.

11.40.-Child sleeping quietly for last two hours. Eyes shut, breathing regularly. Took $1 \mathrm{dr}$. of milk and brandy, tendency to vomit. Pulse 120, resp. 15 ; respiration more sighing.

2nd, 1.20.-Child in same condition. Pulse 116, regular, fair volume. Dark greenish-coloured motion passed, not very offensive. Some solid matter.

10.30.-Child very much better, eyes less sunken, seems in pain at times, no vomiting since 1.35 (vomited immediately after milk). Pulse 120 , respiration 28 , not so sighing in character. Urine passed into bed. Bowels open very slightly.

7.25. -Taken very well to-day, nearly 1 pint. Child much better. Bowels open once. Motion light, unhealthy-looking. Pulse 120, resp. 28. No vomiting.

3rd.-Very much improved, no vomiting, no diarrhœa. Pulse 116, resp. 28 ; eyes much less sunken.

4th.-Child much better; has taken bread and butter, custard pudding, and bread and milk, slept well. Pulse 120, regular. Bowels open once during the night. 5th.-Pulse 112. Child quite well, taken chicken broth, sat up in bed. Bowels not open. Temperature normal.

6th.-Child quite well, bowels not open. Ordered dose of Ol. Ricini. Urine acid, 1012, no albumen.

8th.-Child quite well. Ol. Ricini acted well. Child going home.

Gustave R-, æt. 35.

History.-August 30th.-Left Hamburg on Saturday, August 27th, quite well when starting, rough passage, very sick during the voyage, had diarrhœa, landed at Harwich on Monday morning, August 29th, came to town same morning, seemed fairly well during the day. In 
evening taken ill, had diarrhœea, pain across the back and cramps down the legs, most in right. Diarrhœa continued all night, motions coloured, loose. This morning vomited after medicine given by doctor, no diarrhœa since 11 a.m. this morning. Great pain across the back. Family history.-Child taken ill while at Hamburg, got well again, taken ill again last night.

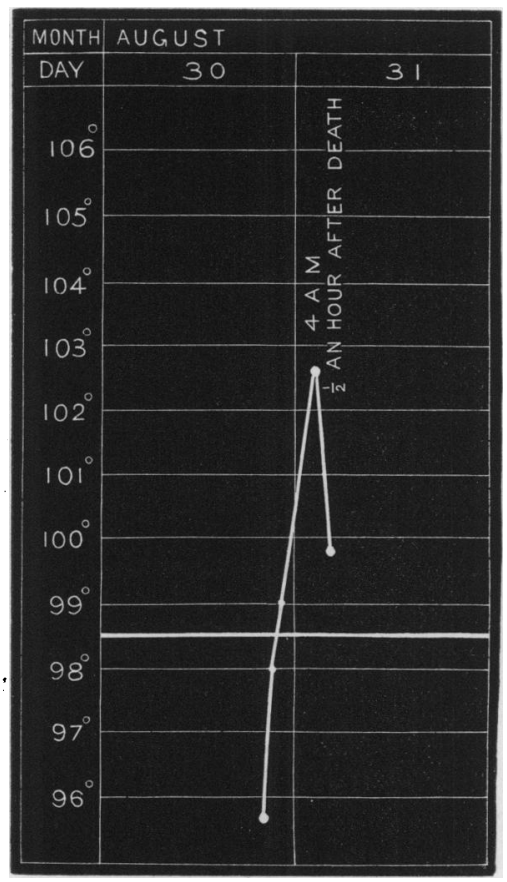

Present condition.-Sallow, grey look, eyes sunken, pupils equal, not contracted, pulse scarcely perceptible at wrist, 148, very feeble. Resp.-Sighing, 56. Temp.$95.8^{\circ}$. Lips dry, very poor colour; tongue moist, not furred. Eyes very much sunken. Cheeks hollow. Chest thickly covered with hair, percussion good all over. Breath sounds natural. Heart.-Apex-beat distinctly felt in fifth interspace. Sounds.-Distinct first sound at 
apex, second sound not heard; sounds very feeble at base. Abdomen not retracted, resonant all over, except hepatic dulness. Bladder not distended above pubes. No tenderness on palpation, not retracted, warm. Arms cold, fingers cold, dusky. Legs fairly warm, feet rather cold, knee-jerks not obtained. Urine passed into bed. Was ordered Liq. Hydrarg. Perchlor. 3ss, Spt. Chlorof. mv, Aq. ad 1 oz. 4tis hr.

10.15.-Very restless, complains of much pain in his back, and some in calves of his legs. Ordered mixture every two hours.

31st, 1.20 a.m.-Still very restless, pulse perceptible at wrist. Passed about $4 \mathrm{oz}$. of urine, B. O. twice, once into bedpan, once into bed. Taken 23 oz. milk, 1 oz. brandy. Hands cold, less pain in back, motions watery, hardly coloured. Pulse 160, resp. 40.

3.30, patient suddenly collapsed. Bowels open just at

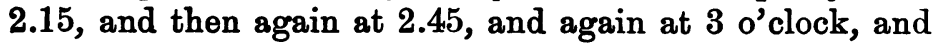
immediately before collapse asked for bedpan. Had taken medicine at 3 , seemed no worse, very restless. Motion light brown colour, no solid matter. Urine highcoloured, fair cloud of albumen, acid.

Post-mortem made nine to ten hours after death.

External appearances.-A medium-sized man. Body moderately well nourished. Abdomen neither retracted nor swollen. Face and eyes sunken. Marked rigor mortis. The blood in the reins of the neck and in the heart was thicker and blacker than usual.

The examination of the head and neck was not allowed. Lungs normal, light and spongy in texture, not engorged. Heart normal, soft clot in the cavities, weight $10 \mathrm{oz}$. Abdomen. Peritoneum.-No peritonitis. External surface of the intestines rather dulled in appearance, and thought by some of those present to be soapy to the touch. General colour rather pale than otherwise, but vessels injected somewhat. The same external appearance throughout all the length of intestine. Stomach.Normal, contained some bilious fluid. Intestines.-In 
upper part of small intestine yellow fluid like the bilecoloured stuff usually found here. Lower down the fluid became paler and thicker (pale greenish yellow), but it was at no point colourless nor " rice-water" in character. The mucous membrane was rather pale in colour with a few reddened patches in lower part of small intestine, but otherwise looked normal. Liver.-Rather small, otherwise normal, weight 44 oz. Spleen.-Small, weight 5 oz., section normal. Abdominal lymphatics.-Only one gland was at all enlarged, and that not greatly, viz. a mesenteric gland near the valve. Kidneys.-Normal in appearance though small, weight $10 \mathrm{oz}$. the pair.

(For report of the discussion on this paper, see 'Proceedings of the Royal Medical and Chirurgical Society,' Third Series, vol. v, p. 122.) 